\title{
Tribological interactions of 3D printed polyurethane and polyamide with water-responsive skin model
}

\author{
Ashish K. KASAR ${ }^{1}$, Ashton CHAN ${ }^{2}$, Victor SHAMANAEV ${ }^{3}$, Pradeep L. MENEZES ${ }^{1, *}$ \\ ${ }^{1}$ Department of Mechanical Engineering, University of Nevada, Reno NV 89557, USA \\ ${ }^{2}$ Department of Mechanical Engineering, New York University, New York 11201, USA \\ ${ }^{3}$ Department of Mechanical Engineering, University of Nevada, Las Vegas NV 89154, USA \\ Received: 04 July 2020 / Revised: 16 October 2020 / Accepted: 10 November 2020 \\ (C) The author(s) 2020 .
}

\begin{abstract}
D printing in the textile and fashion industry is a new emerging technology. Applications of 3D printing for designing clothes and other wearable accessories require tribological and biological understanding of $3 \mathrm{D}$ printing plastics against the complex human skin to mitigate skin-friction related ailments such as calluses and blisters. This study provides tribological insight in search of an optimal 3D printable material that has minimal friction against the skin. Two low friction 3D printable materials, thermoplastic polyurethane (TPU) and polyamide (TPA) were chosen and tribological testing was carried out against a water responsive skin model. The skin model was synthesized using a gelatine based model made with cotton and crosslinked with glutaraldehyde. Tribological testing of TPU/TPA against the skin model in dry and wet conditions were made. The higher coefficient of friction (COF) was observed in the wet condition compared to the dry condition. To overcome the higher friction, TPA/TPU-sodium polyacrylate composites were prepared by heat pressing that significantly reduced COF of TPU and TPA by 40\% and 75\%, respectively, in wet conditions.
\end{abstract}

Keywords: coefficient of friction (COF); skin model; thermoplastic polyurethane; thermoplastic polyamide

\section{Introduction}

3D printing is an advancing technology in the field of textile and fashion industry. The emerging technical possibilities of 3D printing have led to the development of fully 3D printed clothes and foot wares with intricate features using flexible plastics [1, 2]. 3D printing also enables to design clothes and shoes suitable for individual body type. The other important benefits of 3D printing are customizable dynamic surface material, ease of manufacturing, and rapid prototype for testing the wearable accessories and gadgets. However, pure 3D printed clothes, sports accessories, and other garments are still far away compared to conventional clothing materials. One of the main challenges is the compatibility issue of plastics with human skin. Human skin is a complex surface, requires investigation for better usage of these materials by avoiding any skin disorders or bruises on the skin [3,4]. Especially, designing of the cloth materials are challenging due to varying behavior of skin because of sweating or the presence of moisture.

The tribological interaction of human skin depends on many factors. It depends on several biological factors such as age, gender, health conditions, anatomical region, or hydration level $[5,6]$. The frictional behavior of the human skin is also influenced by the amount of water in the form of sweat or moisture between the skin and contacting materials [6]. The human skin is comprised of three layers: i) Epidermis, ii) Dermis, and iii) Hypodermis [7]. The top layer Epidermis consists of stratum corneum, which is considered as a rough and stiff material under atmospheric conditions. However, moisture or sweating leads to smoothening

* Corresponding author: Pradeep L. MENEZES, E-mail: pmenezes@unr.edu 
and softening of the skin and causes a high coefficient of friction (COF) $[3,8,9]$. Other than these biological factors, the tribological interactions of human skin are also dependent on the counter materials properties, such as roughness, mechanical properties, and hydrophobicity/hydrophilicity nature [6].

In order to find the optimal counter material, which is also $3 \mathrm{D}$ printable using the fusion deposition modeling (FDM) technique, the materials range goes down to thermoplastic polymers. Polymers selected for counter material should have specific properties, including a low COF both at hydrated and unhydrated conditions, nontoxic, and easily obtainable. The materials that met most of the selective criteria are narrowed down to thermoplastic polyurethane (TPU) and Nylon, also known as thermoplastic polyamide (TPA). Because, both the materials fabricated conventional method such as injection molding, have shown the low COF against human skin that can boost application of 3D printed TPA and TPU [10,11]. However, TPU and TPA do not have the waterabsorbing capacity in the presence of moisture or sweating. 3D printing technology is advantageous to overcome this limitation by modifying the structure of the polymer counter materials. Modification of the structure is carried out by adjusting the percentage fill density and interweaving a water-absorbent polymer material in the polymer samples. The material for the interwoven absorbent layer requires two specific properties: nontoxic and excellent hydrophilic capabilities. The material that matches this requirement is sodium polyacrylate $[12,13]$.

In this work, in-vivo tribological testing is avoided because it involves several biological factors that can be challenging and require many test trials. A water responsive skin model, developed by Dąbrowska et al. [14], is used. The 3D printed TPA, TPU, and their composite with sodium polyacrylate are investigated against the skin model and evaluated the performance in terms of COF in wet and dry conditions.

\section{Methods and materials}

\subsection{D printing of polyurethane and polyimide}

Printing of thermoplastic polyamide (TPA) and thermoplastic polyurethane (TPU) has been done using FDM process by a Prusa i3MK3 printer. The TPA and TPU filament of $1.75 \mathrm{~mm}$ were purchased from MatterHackers-3D printing supplies. The nozzle size of the printer was $0.4 \mathrm{~mm}$. Printing of flexible material is tedious, and it can be achieved by optimizing various $3 \mathrm{D}$ printing parameters, such as nozzle temperature, extrusion rate, layer height, and print head speed during the prerequisite testing campaign. The infill printing speed was set to be $200 \mathrm{~mm} / \mathrm{s}$ for rapid printing with a layer height of $0.1 \mathrm{~mm}$. For the TPA printing, the nozzle temperature and build plate temperatures were set to be 260 and $85^{\circ} \mathrm{C}$, respectively. Whereas, lower nozzle $\left(239^{\circ} \mathrm{C}\right)$ and build plate $\left(50{ }^{\circ} \mathrm{C}\right)$ temperature were used for TPU due to its lower melting point. The final TPA and TPU samples with $2 \mathrm{~mm}$ thick were printed in rectilinear pattern with $80 \%$ infill density.

The composite of polymer (TPA/TPU) and sodium polyacrylate was prepared by filling the 1-2 $\mathrm{g}$ of sodium polyacrylate crystals between two samples of 3D printed polymers followed by heat pressing at $170{ }^{\circ} \mathrm{C}$. The heat pressing at high temperature was carried out to retain the sodium polyacrylate crystals by fusing the thermoplastic samples. The heat pressing at high temperature also altered the roughness of the composite surfaces. Also, TPA and TPU samples without sodium polyacrylate were also prepared by heat pressing for comparison which are designated as heat pressed base polymers.

\subsection{Synthesis of the skin model}

In order to understand the tribology of the human skin, a water responsive, gelatine-based skin model developed by Dąbrowska et al. is used [14], and the schematic of the synthesis process is shown in Fig. 1. The fabrication of this skin model was done by stirring $50 \mathrm{~mL}$ of a $10 \mathrm{wt} \%$ gelatine (type A, bloom no 300, Sigma Aldrich) solution in distilled water at $60{ }^{\circ} \mathrm{C}$ for $2 \mathrm{~h}$. The solution was placed in a large beaker of water and covered with parafilm to prevent the loss of heat in the solution. Then, pieces of cheesecloth (Grade 90) were placed onto a glass sheet and the resulting solution was spread onto the single layer of cloth pieces manually by using a bar coater. The spreading of the solution was done three times and then the material rested for $24 \mathrm{~h}$ to dry. The resulting 


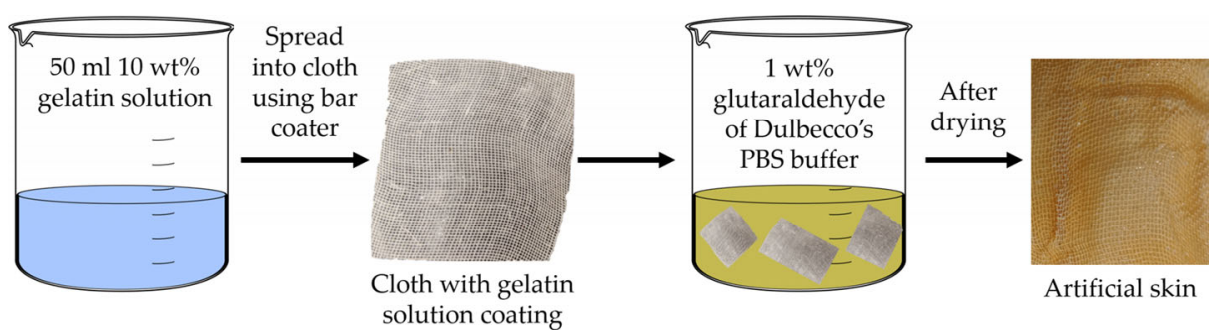

Fig. 1 Schematic of the synthesis of skin model.

composite material was placed in $200 \mathrm{~mL}$ of $1 \mathrm{wt} \%$ solution of glutaraldehyde inside of Dulbecco's PBS buffer and mixed at low speed for $24 \mathrm{~h}$. The composite material was then extracted and covered in four layers of paper towels and $4 \mathrm{~kg}$ of weight. The towels were replaced two times a day for three days.

\subsection{Contact angle and surface roughness measurement}

The static contact angle measurements on the polymer surfaces were carried out using rame-hart contact angle Goniometer (ram-hart, New Jersey, NJ, USA) by sessile drop method. Distilled water droplets of $5 \mu \mathrm{l}$ were placed on the TPA/TPU surfaces and at least five readings were taken for each polymer. The difference between left and right contact angles was less than $2^{\circ}$. The contact angle was measured on the 3D printed TPA and TPU with $100 \%$ fill structure (no porosity), which was aimed to characterize the inherent hydrophilic property of these polymers. The skin model, TPA and TPU surfaces were observed at 10× under optical profilometer (Rtech-instruments) and Keyence 3D Digital Microscope VHX-5000 to measure their surface roughness.

\subsection{Tribological testing}

Sliding tests were performed using Rtech-Tribometer, where 3D printed TPA and TPU were slid against the skin model. During sliding, the skin model was wrapped on a steel ball of $6.35 \mathrm{~mm}$ diameter to measure the frictional interactions against 3D printed polymers, as shown in Fig. 2. The dry sliding tests were carried out at room temperature $\left(\sim 25{ }^{\circ} \mathrm{C}\right)$ and $40 \%-50 \%$ relative humidity. Wet sliding tests were conducted to simulate the sweating condition by spreading water on the polymer surfaces. For wet sliding tests, the optimized water content $(20 \mu \mathrm{L})$ was placed on the 3D printed polymer using a micro syringe. The linear sliding tests were conducted for a distance of $15 \mathrm{~mm}$ at a normal load of $4 \mathrm{~N}$ and skin model wrapped on the steel ball moved was sliding with a velocity of $1 \mathrm{~mm} / \mathrm{s}$ over the stationary 3D printed polymer surfaces. The lower load $(<10 \mathrm{~N})$ and lower velocity are preferable to simulate the relative motion between any fabric and human skin, as indicated in earlier studies [15-17]. In the present study, the estimated contact pressure is $\sim 300 \mathrm{kPa}$, which is an order of magnitude higher than the contact pressure reported in previous studies of skin-fabric contacts $[4,17]$. The tribological tests conducted three times at each condition were to ensure the repeatability of the test. During the tests, the frictional force was recorded that used to calculate the COF. The linear sliding tests were conducted across the rectilinear pattern.

Composite of TPA/TPU and sodium polyacrylate were prepared by filling the crystals of sodium polyacrylate between two 3D printed samples of TPA/TPU followed by heat pressing. The heat pressing resulted in capturing the sodium polyacrylate crystals between the $80 \%$ infill polymer layers. Wet sliding tests were conducted on the prepared composites to measure the friction while absorbing water. Prior

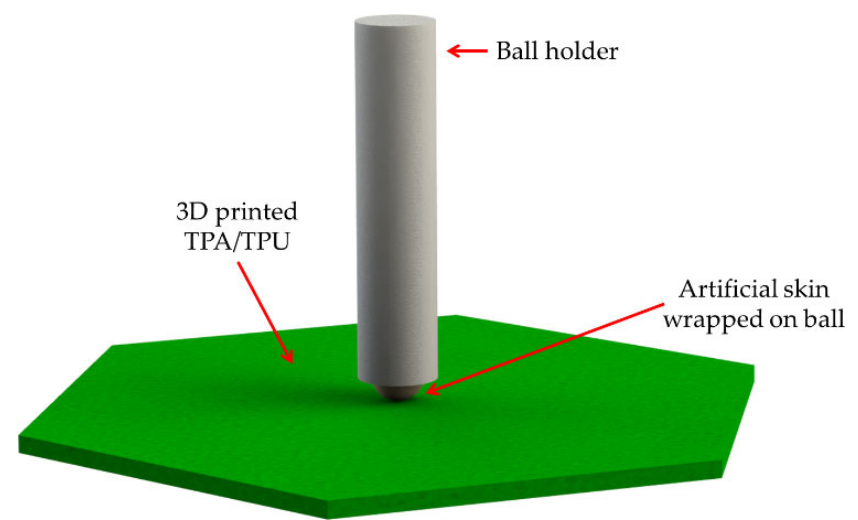

Fig. 2 Schematic for sliding test setup to measure friction. 
to sliding tests, the heat pressed surfaces were characterized using an optical profilometer to measure the change in roughness due to heat press.

\section{Results and discussion}

\subsection{Surface characteristics and contact angle measurement}

The skin model synthesized using cheesecloth is shown in Fig. 3(a). Figure 3(b) shows the optical image of the skin model using a 3D optical microscope. The threads of the cotton cloths can be clearly seen. However, the gelatin can not be seen under white light due to its translucent nature. The 3D profile of the skin model shown in Figs. 3(c) and 4 confirms the presence of gelatin between threads by showing the uniform layer. Figure 4 obtained by an optical profilometer, is used to calculate the thickness of the
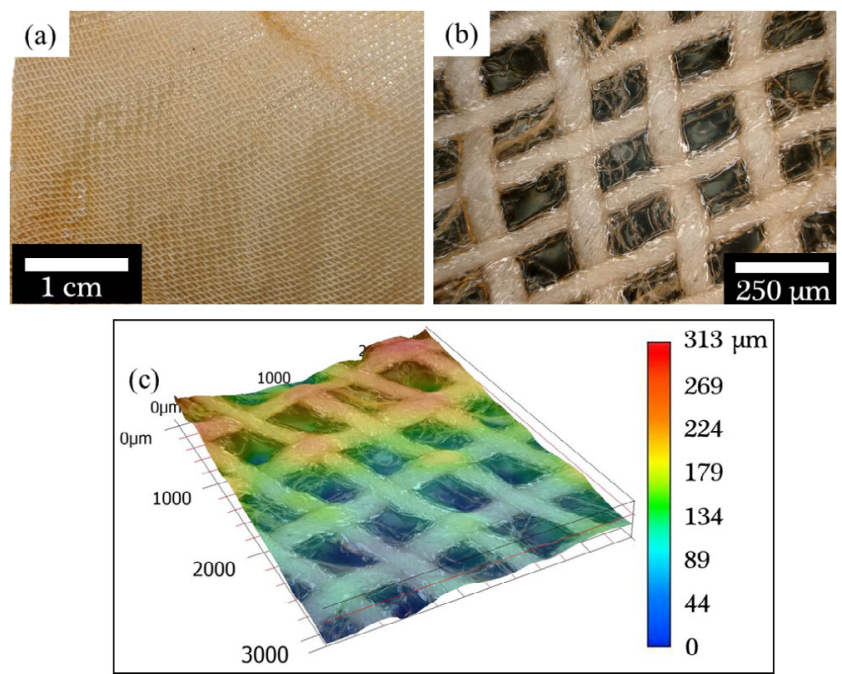

Fig. 3 (a) Synthesized water responsive skin model; (b) optical micrograph; and (c) 3D image of the skin model using 3D optical microscope. skin model. The flattened skin model was stick to the flat surface, and 3D scans were recorded across the edge of the skin model. The difference in height from the skin model to the flat surface is used to calculate the thickness of the skin model that is observed to be $57.5 \pm 2.3 \mu \mathrm{m}$. Whereas the surface roughness of the skin is found to be $S_{a} \sim 4.2 \mu \mathrm{m}$.

TPA and TPU surfaces are shown in Figs. 5 and 6, respectively. The rectilinear pattern can be clearly seen on the surfaces. The optical micrographs of TPA and TPU (Figs. 5(b) and 6(b)) are evident of porosity in the samples due to $80 \%$ fill during $3 \mathrm{D}$ printing. The 3D profiles using optical profilometer are shown in Figs. 5(c) and 6(c), are used to calculate the roughness of the 3D printed surfaces. The surface roughness of the TPA surface is $S_{a}=50.7 \pm 4.3 \mu \mathrm{m}$ and for TPU surface is $S_{a}=16.42 \pm 4.3 \mu \mathrm{m}$. The lower surface roughness of the TPU compared to TPA was due to its higher flowability, which helps to settle down and weld with adjacent layers during printing.

Hydrophobic/hydrophilic nature of the material against human skin is an essential criterion due to sweating. The measured contact angle for TPA is $64.1^{\circ} \pm 2.0^{\circ}$, and for TPU, it is $60.2^{\circ} \pm 2.3^{\circ}$ are shown in Figs. 7(a) and 7(b), respectively. The observed contact angles data are comparable to the data mentioned in with the Refs. [18, 19], and contact angle ranges also suggest that the water droplet will not stick to the polymer surfaces.

\section{$3.2 \mathrm{COF}$}

The COF for as printed TPA and TPU samples against the skin model in dry and wet conditions are shown in Figs. 8(a) and 8(b), respectively. For both polymers, friction increased in the wet condition compared to dry condition, it is due to the water-responsive skin

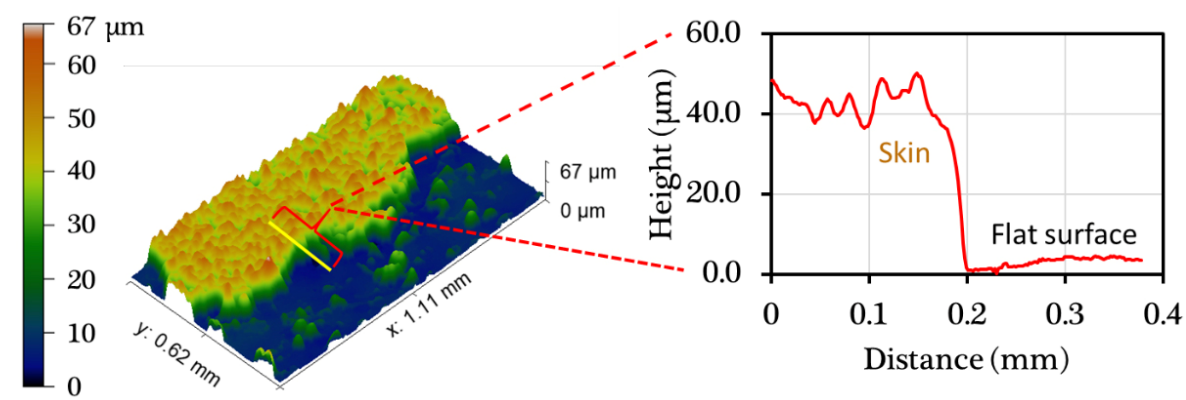

Fig. 4 Skin height calculation. 


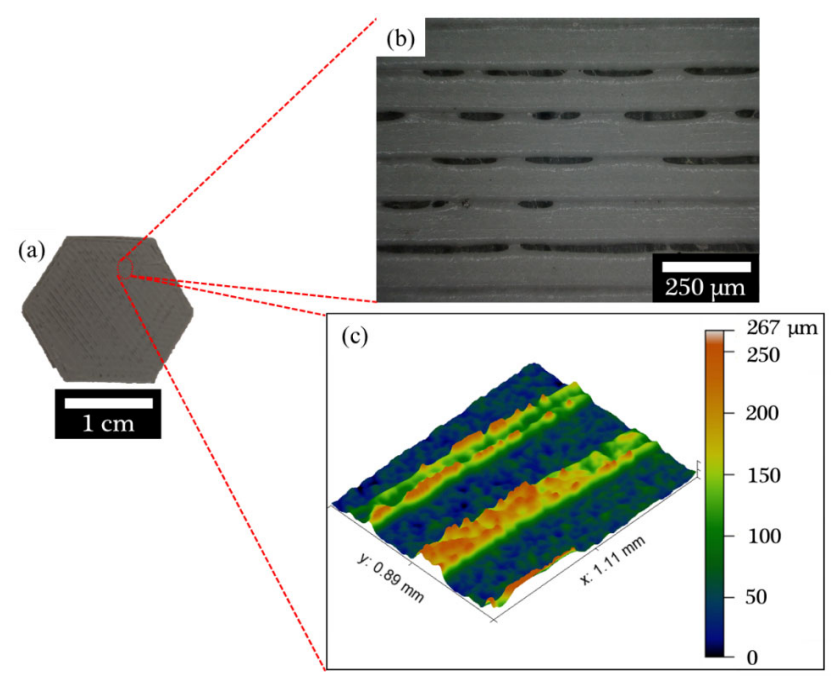

Fig. 5 (a) 3D printed TPA specimen; (b) optical image; and (c) surface profile of the TPA.

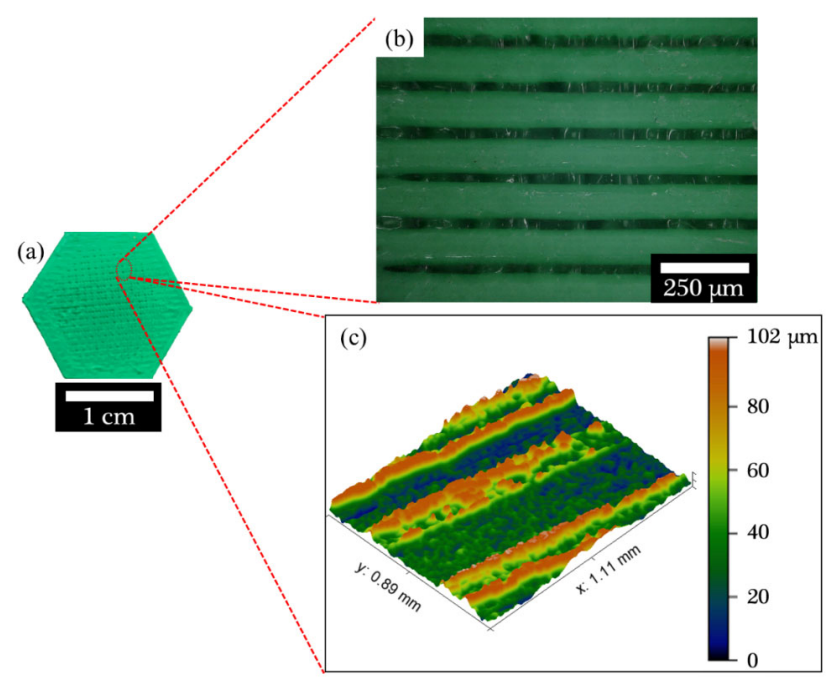

Fig. 6 (a) 3D printed TPU specimen; (b) optical image; and (c) surface profile of the TPU.

model. The similar behavior of this skin model is also indicated against worsted wool cloth [14]. It also confirms the skin model replicates the nature of the human skin well in moist or in sweat condition and the results are in accordance with the adhesion theory of human skin friction $[9,20]$.

In addition, COF values fluctuate significantly in dry condition compared to the wet condition. The observed fluctuations in dry condition were possibly due to the 3D printed patterns. While in wet condition, the fluctuations are not present because of induced softening in the skin model and the softening behavior is also shown in human skin [20].

The average COF observed for polymer-sodium polyacrylate composites after heat pressing is shown in Fig. 9. Dry and wet COF values in Fig. 9 show the COF for TPA and TPU after heat pressing without the addition of sodium polyacrylate. By comparing Figs. 8 and 9, it can be clearly seen that the COF decreased after heat pressing the polymer. For example, COF for TPA in dry conditions reduced from 0.24 to 0.1 due to heat pressing. The heat pressing process leads to a change in the surface morphology of the polymer and reduces the surface roughness. The surface roughness $\left(\mathrm{S}_{\mathrm{a}}\right)$ of the TPA and TPU after heat pressing was reduced to $6.7 \pm 0.3$ and $3.5 \pm 0.4 \mu \mathrm{m}$, respectively with and without sodium polyacrylate. It is expected that the reduction in surface roughness reduces COF. It can also be deduced that the addition of sodium polyacrylate did not affect the surface roughness, the heat pressing is the main reason to reduce the roughness. From Figs. 8 and 9, it can also be seen that the TPU has higher COF than the TPA in both dry and wet condition. The higher COF of TPU could be due to its elastomer characteristics that increase adhesion friction.

As discussed previously, wetting leads to softening of the skin model that increases the COF. The same behavior is also observed after heat pressing of two polymer samples. The COF for TPA is increased from $0.1 \pm 0.03$ to $0.19 \pm 0.02$ in wet condition, and for TPU,
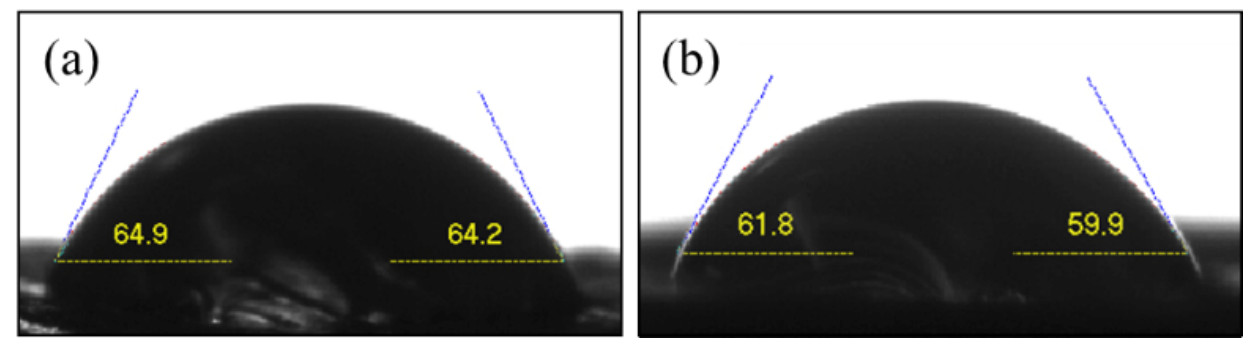

Fig. 7 Contact angle measurement on (a) TPA and (b) TPU specimen. 
(a)

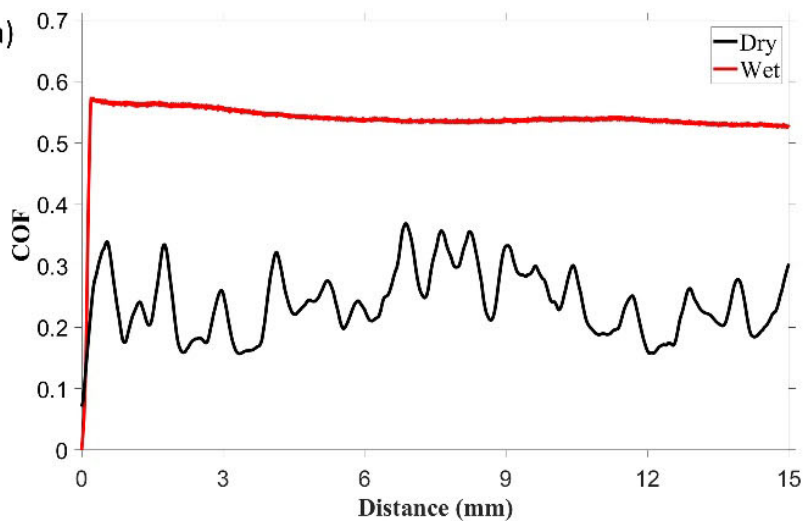

(b) 0.7

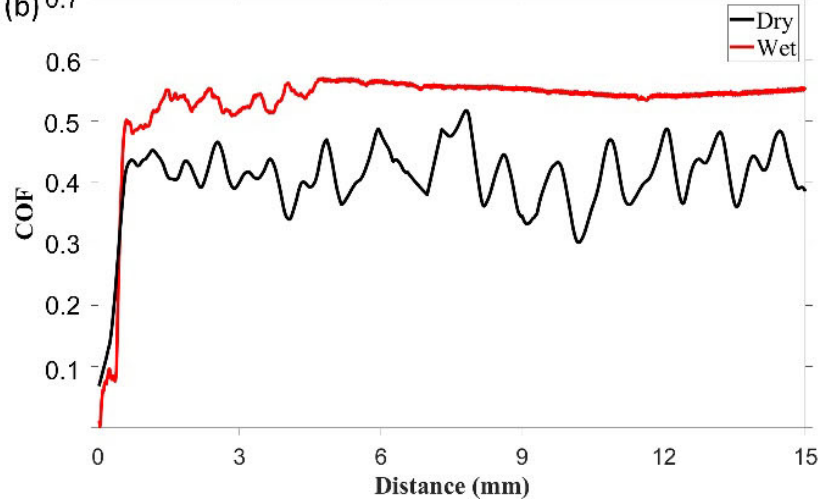

Fig. 8 COF of as printed (a) TPA and (b) TPU against the skin model.

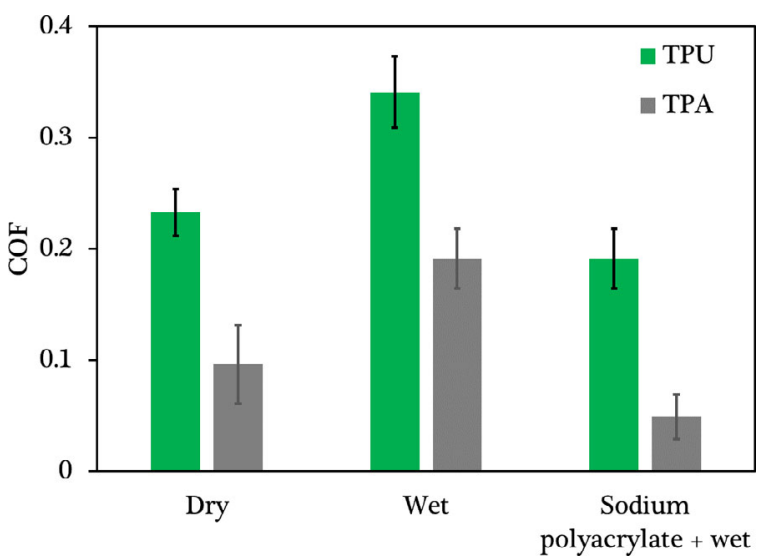

Fig. 9 COF variation of heat pressed TPA; TPU; and their composites with sodium polyacrylate against the skin model.

the COF is increased from $0.23 \pm 0.02$ to $0.34 \pm 0.03$. The composite polymer samples with sodium polyacrylate were tested immediately after applying water on the surfaces. The COF for the composite was drastically reduced compared to heat pressed base polymers (TPA and TPU) in wet condition. The TPA composite showed $\sim 74 \%$ decrease in COF whereas the TPU composite showed $\sim 44 \%$ decrease in COF with respect to their heat pressed base polymers.

As shown earlier the softening of the skin model in wet condition resulted in higher COF. However, the COF for the composites (wet condition) was lesser than the COF of as printed base polymers (TPA and TPU) in dry conditions, which can be elucidated by comparing the dry COF shown in Fig. 8 and COF of the composite in Fig. 9. For example, the average COF of TPU composite in wet condition (Fig. 9) is 0.19 which is lesser than the average COF (0.4) of as printed TPU (Fig. 8) in dry condition. Similar trend can be seen for TPA. The reduction of friction for the polymer composite was observed because of two reasons: i) lower roughness obtained through heat press and ii) absorbing water by the sodium polyacrylate crystals. The 3D printed structure is designed with $80 \%$ fill structure that enables the water to enter inside the samples through connecting pores. Then the sodium polyacrylate crystals captured between polymer samples, absorb the water.

\section{Conclusions}

The evidence of the coefficient of friction (COF) increasing in wet condition shows that the synthesized skin model behaves similarly to real human skin. This effect is due to adhesion properties of the skin model. Whereas the observed COF fluctuation in dry condition due to 3D printed patterns suggests that the printing pattern should be considered while designing the $3 \mathrm{D}$ printed fabric/accessories to achieve the desired comfort. The results also show that heat pressing of polyamide (TPA) and thermoplastic polyurethane (TPU) provides lower COF values both in dry and wet conditions by lowering the surface roughness. This change in surface characteristics suggests the possibilities of post-treatment on 3D printed objects to control the tribological properties.

The TPA and TPU composites filled with waterabsorbing polymer, sodium polyacrylate successfully reduced the COF by absorbing water quickly. Due to the water-absorbing capacity of sodium polyacrylate, water was not in contact with the skin model that avoided adhesion friction. Further research should 
delve into a way to mitigate moisture on the skin to improve clothing and skin interaction. The present study highlights the design of polymers that can be 3D printed and have low COF for better comfortability. An issue presents within human skin polymer interaction such as the generation of sweat can be solved using material such as sodium polyacrylate to absorb the moisture directly. Another possible solution to reduce the sweat generation is through an alternative form of cooling/heat dissipation. It is believed that the future clothing needs to integrate some method of increased water absorption or heat dissipation for suitable skin to clothing contact. The 3D printed thermoplastics such as TPU or TPA could be interwoven into normal clothing for decreased COF and increased comfortability, while sodium polyacrylate could absorb any water and keep the individual cool. The 3D printing technique has advantages over the conventional manufacturing such as, control over the pore size, volume, intricate designs and easily alteration in design based on geometric requirements.

\section{Acknowledgements}

The authors acknowledge the financial support from US National Science Foundation (1852578) and startup funding from the Department of Mechanical Engineering at the University of Nevada, Reno.

Open Access This article is licensed under a Creative Commons Attribution 4.0 International License, which permits use, sharing, adaptation, distribution and reproduction in any medium or format, as long as you give appropriate credit to the original author(s) and the source, provide a link to the Creative Commons licence, and indicate if changes were made.

The images or other third party material in this article are included in the article's Creative Commons licence, unless indicated otherwise in a credit line to the material. If material is not included in the article's Creative Commons licence and your intended use is not permitted by statutory regulation or exceeds the permitted use, you will need to obtain permission directly from the copyright holder.

To view a copy of this licence, visit http://creativecommons.org/licenses/by/4.0/.

\section{References}

[1] 3D printing in textile industry: Clothes and textile. https://www.sculpteo.com/en/applications/textile-industry/, 2020.

[2] How 3D printing in the textile industry is leading into a new era. https://www.sculpteo.com/en/applications/textile-industry/, 2020.

[3] Derler S, Schrade U, Gerhardt L-C. Tribology of human skin and mechanical skin equivalents in contact with textiles. Wear 263: 1112-1116 (2007)

[4] Gerhardt L C, Mattle N, Schrade G, Spencer N, Derler S. Study of skin-fabric interactions of relevance to decubitus: Friction and contact-pressure measurements. Skin Res Technol 14: 77-88 (2008)

[5] Dąbrowska A, Rotaru G M, Derler S, Spano F, Camenzind M, Annaheim S, et al. Materials used to simulate physical properties of human skin. Skin Res Technol 22: 3-14 (2016)

[6] Derler S, Gerhardt L-C. Tribology of skin: Review and analysis of experimental results for the friction coefficient of human skin. Tribol Lett 45: 1-27 (2012)

[7] Yousef H, Alhajj M, Sharma S. Anatomy, Skin (Integument), Epidermis. Treasure Island (USA): StatPearls Publishing, 2017.

[8] Gerhardt L-C, Strässle V, Lenz A, Spencer N, Derler S. Influence of epidermal hydration on the friction of human skin against textiles. $J R$ Soc Interface 5: 1317-1328 (2008)

[9] Hendriks C, Franklin S. Influence of surface roughness, material and climate conditions on the friction of human skin. Tribol Lett 37: 361-373 (2010)

[10] Zhang M, Mak A. In vivo friction properties of human skin. Prosthet Orthot Int 23: 135-141 (1999)

[11] Vilhena L, Ramalho A. Friction of human skin against different fabrics for medical use. Lubricants 4: 6 (2016)

[12] Ogawa I, Yamano H, Miyagawa K. Rate of swelling of sodium polyacrylate. J Appl Polym Sci 47: 217-222 (1993)

[13] Park J H, Kim D. Preparation and characterization of waterswellable natural rubbers. J Appl Polym Sci 80: 115-121 (2001)

[14] Dąbrowska A, Rotaru G M, Spano F, Affolter C, Fortunato G, Lehmann S, et al. A water-responsive, gelatine-based human skin model. Tribol Int 113: 316-322 (2017)

[15] Tang W, Ge S-r, Zhu H, Cao X-c, Li N. The influence of normal load and sliding speed on frictional properties of skin. J Bionic Eng 5: 33-38 (2008)

[16] Veijgen N, Masen M A, van der Heide E. A novel approach to measuring the frictional behaviour of human skin in vivo. Tribol Int 54: 38-41 (2012)

[17] Cottenden D J, Cottenden A M. A study of friction mechanisms between a surrogate skin (Lorica soft) and nonwoven fabrics. J Mech Behav Biomed mate 28: 410-426 (2013) 
[18] Li W, Liu J, Hao C, Jiang K, Xu D, Wang D. Interaction of thermoplastic polyurethane with polyamide 1212 and its influence on the thermal and mechanical properties of TPU/PA1212 blends. Polym Eng Sci 48: 249-256 (2008)

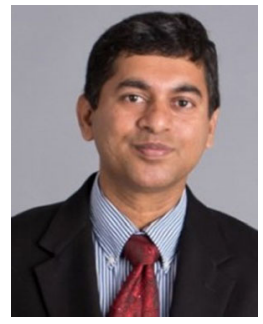

Pradeep L. MENEZES. He received his Ph.D. in materials engineering from Indian Institute of Science, Bangalore India in 2008. Later he joined as post-doctoral researcher at University of Pittsburgh and then

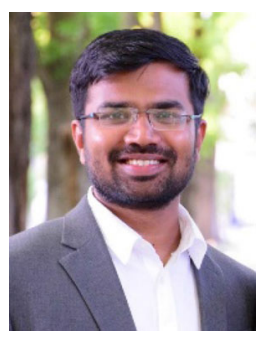

Ashish K. KASAR. He is a Ph.D. student in mechanical engineering at University of Nevada, Reno. Prior to $\mathrm{Ph} . \mathrm{D}$., he received his bachelor's degree in metallurgical engineering
[19] Król P, Król B. Surface free energy of polyurethane coatings with improved hydrophobicity. Colloid Polym Sci 290: 879-893 (2012)

[20] Adams M J, Briscoe B J, Johnson S A. Friction and lubrication of human skin. Tribol lett 26: 239-253 (2007)

University of Wisconsin-Milwaukee. He is an assistant professor in the Mechanical Engineering Department at University of Nevada Reno since 2015. His research interests are tribology, surface engineering, manufacturing, and materials science.

from National Institute of Technology, Raipur and master's degree in materials science and engineering from Indian Institute of Technology, Kanpur. His research interests are tribology, tribocorrosion, and materials science. 